\title{
BMJ Open Management of chronic neuropathic pain: a protocol for a multiple treatment comparison meta-analysis of randomised controlled trials
}

\author{
Sohail M Mulla, ${ }^{1,2}$ D Norman Buckley, ${ }^{3,4}$ Dwight E Moulin, ${ }^{5}$ Rachel Couban, ${ }^{4}$ \\ Zain Izhar, ${ }^{1}$ Arnav Agarwal, ${ }^{1}$ Akbar Panju, ${ }^{4,6}$ Li Wang, ${ }^{3}$ Sun Makosso Kallyth, ${ }^{4}$ \\ Alparslan Turan, ${ }^{7}$ Victor M Montori, ${ }^{8}$ Daniel I Sessler, ${ }^{7}$ Lehana Thabane, ${ }^{1,3,9}$ \\ Gordon H Guyatt, ${ }^{1,6}$ Jason W Busse ${ }^{1,3,4}$
}

To cite: Mulla SM,

Buckley DN, Moulin DE, et al. Management of chronic neuropathic pain: a protocol for a multiple treatment comparison meta-analysis of randomised controlled trials. BMJ Open 2014:4:e006112. doi:10.1136/bmjopen-2014006112

- Prepublication history and additional material is available. To view please visit the journal (http://dx.doi.org/ 10.1136/bmjopen-2014006112).

Received 15 July 2014 Revised 14 October 2014 Accepted 31 October 2014

CrossMark

For numbered affiliations see end of article.

Correspondence to

Sohail M Mulla;

mullasm@mcmaster.ca

\section{ABSTRACT}

Introduction: Chronic neuropathic pain is associated with reduced health-related quality of life and substantial socioeconomic costs. Current research addressing management of chronic neuropathic pain is limited. No review has evaluated all interventional studies for chronic neuropathic pain, which limits attempts to make inferences regarding the relative effectiveness of treatments.

Methods and analysis: We will conduct a systematic review of all randomised controlled trials evaluating therapies for chronic neuropathic pain. We will identify eligible trials, in any language, by a systematic search of CINAHL, EMBASE, MEDLINE, AMED, HealthSTAR, DARE, PsychINFO and the Cochrane Central Registry of Controlled Trials. Eligible trials will be: (1) enrol patients presenting with chronic neuropathic pain, and (2) randomise patients to alternative interventions (pharmacological or non-pharmacological) or an intervention and a control arm. Pairs of reviewers will, independently and in duplicate, screen titles and abstracts of identified citations, review the full texts of potentially eligible trials and extract information from eligible trials. We will use a modified Cochrane instrument to evaluate risk of bias of eligible studies, recommendations from the Initiative on Methods, Measurement, and Pain Assessment in Clinical Trials (IMMPACT) to inform the outcomes we will collect, and the Grading of Recommendations Assessment, Development and Evaluation (GRADE) system to evaluate our confidence in treatment effects. When possible, we will conduct: (1) in direct comparisons, a random-effects meta-analysis to establish the effect of reported therapies on patient-important outcomes; and (2) a multiple treatment comparison meta-analysis within a Bayesian framework to assess the relative effects of treatments. We will define a priori hypotheses to explain heterogeneity between studies, and conduct meta-regression and subgroup analyses consistent with the current best practices.

Ethics and Dissemination: We do not require ethics approval for our proposed review. We will disseminate our findings through peer-reviewed publications and conference presentations.

\section{Strengths and limitations of this study}

- Our broad study eligibility criteria will allow us to generate more precise estimates of treatment effects, thus increasing generalisability of our results.

- We will use the Grading of Recommendations Assessment, Development and Evaluation (GRADE) approach to evaluate our confidence in treatment effects, and the IMMPACT guidelines to inform the outcomes we will collect. No existing review on the topic has done so.

- We will ensure interpretability by presenting risk differences and measures of relative effect for all outcomes reported, and by presenting our findings with GRADE evidence profiles. No existing review on the topic has done so.

- Our results will be limited by possible shortcomings of the primary studies.

Trial registration number: PROSPERO (CRD42014009212).

\section{BACKGROUND}

Chronic neuropathic pain is defined as "pain arising as a direct consequence of a lesion or disease affecting the somatosensory system."1 It may be classified as central or peripheral, depending on the site of the lesion. ${ }^{2}$ Among the causes of chronic neuropathic pain are metabolic disease (eg, diabetes), infection (eg, shingles), trauma (eg, spinal cord injury) and autoimmune disease (eg, multiple sclerosis). ${ }^{3-5}$ The pain may be spontaneous or evoked in response to physical stimuli. The latter may manifest as increased sensitivity to pain (hyperalgesia) or as a painful response to a stimulus that would not normally be painful (allodynia). ${ }^{46}$ 
Chronic neuropathic pain is common worldwide, affecting $7 \%$ to $10 \%$ of the general population. ${ }^{7}$ It is associated with depression, anxiety and sleep disturbances, and patients with chronic neuropathic pain experience lower health-related quality of life than the general population. ${ }^{8-11}$

Chronic neuropathic pain is associated with substantial economic burden. Tarride et $a l^{12}$ estimated that managing a Canadian patient with chronic neuropathic pain over a 3-month period costs an average of $\$ 2567$, of which $52 \%$ are direct costs, for example, cost of physicians, diagnostic tests and surgical procedures. Others report that people suffering from chronic neuropathic pain generate medical costs that are three times greater than those not living with pain. ${ }^{11}{ }^{13}$ In the USA alone, almost $\$ 40$ billion annually in healthcare, disability and related costs is attributed to chronic neuropathic pain. ${ }^{4}$

The underlying mechanisms of chronic neuropathic pain are poorly understood, which complicates management. Non-pharmacological and pharmacological treatments are currently used. A limited number of systematic reviews focus on non-pharmacological options, including electrical nerve stimulation, ${ }^{14}$ acupuncture ${ }^{15}{ }^{16}$ and cognitive behavioural therapy. ${ }^{17}$ Most report pharmacological treatments for chronic neuropathic pain, including antidepressants, ${ }^{18}$ anticonvulsants ${ }^{19}$ and opioid analgaesics. $^{20}$

However, significant gaps remain. For example, randomised controlled trials (RCTs) exploring treatment for chronic neuropathic pain often compare pharmacological treatments against placebo and seldom against each other. Consequently, there are few direct comparisons among treatments. A recent systematic review found that among 131 RCTs published between 1969 and 2007, addressing painful diabetic neuropathy and postherpetic neuralgia, both common types of peripheral neuropathic pain, only 25 studies (19\%) compared drugs directly against each other. ${ }^{21}$

No review to date has systematically evaluated all evidence for management of chronic neuropathic pain; existing reviews focus on select therapies ${ }^{18} 2022-46$ or specific syndromes. ${ }^{47-57}$ Additionally, risk of bias assessment of studies included in existing reviews has been variable, and authors often depended on instruments that have been criticised for being overly simplistic (eg, Jadad system) and/or assessed risk of bias on a per-study basis rather than overall for reported outcome. ${ }^{58} 59$ Furthermore, strategies to identify studies have been limited, as authors used few search terms, did not search major literature databases, and/or did not consider foreign language studies-an approach that would have excluded $12 \%$ of eligible trials in a systematic review of another chronic pain syndrome. ${ }^{60}$ As well, none of the reviews employ the Grading of Recommendations Assessment, Development and Evaluation (GRADE) approach to evaluate the confidence in effect estimates (quality of evidence) for reported outcomes. And, finally, none of the existing reviews facilitate interpretability, for instance, by presenting results in terms of minimally important differences (MID).

The limitation of previous works suggests the need for a new systematic review to be conducted using state-of-the-art methodology to inform evidence-based management of chronic neuropathic pain. We thus plan a systematic review and multiple treatment comparison meta-analysis of therapies for chronic neuropathic pain.

\section{METHODS}

\section{Standardised reporting}

Our paper will conform to the Preferred Reporting Items for Systematic Reviews and Meta-Analyses (PRISMA) guidelines for reporting systematic reviews of RCTs.

\section{Protocol registration}

Our protocol is registered on PROSPERO (registration number: CRD42014009212).

\section{Search strategy}

We will identify relevant RCTs, in any language, by a systematic search of CINAHL, EMBASE, MEDLINE, AMED, HealthSTAR, DARE, PsychINFO, PapersFirst, ProceedingsFirst and the Cochrane Central Registry of Controlled Trials, from the inception of each database. Our search will be refined for individual databases by a highly experienced medical librarian (RC; see online supplementary appendix 1 , which is a proposed search strategy for MEDLINE). Reviewers will scan the bibliographies of all retrieved trials and other relevant publications, including reviews and meta-analyses, for additional relevant articles.

\section{Eligibility criteria and their application to potentially eligible articles}

Using standardised forms, reviewers trained in health research methodology will work in pairs to screen, independently and in duplicate, titles and abstracts of identified citations, and acquire the full-text publication of articles that both reviewers judge as potentially eligible. Using a standardised form, the same reviewer teams will independently apply eligibility criteria to the full text of potentially eligible trials. We will measure agreement between reviewers to assess the reliability of full-text review using the guidelines proposed by Landis and Koch. ${ }^{61}$ Specifically, we will calculate $\kappa$ values, and interpret them using the following thresholds: $<0.20$ as slight agreement, $0.21-0.40$ as fair agreement, $0.41-0.60$ as moderate agreement, $0.61-0.80$ as substantial agreement and $>0.80$ as almost perfect agreement. Eligible trials will be: (1) enrol patients presenting with chronic neuropathic pain (see online supplementary appendix 2 for lists of all syndromes we are studying) and (2) randomise patients to alternative interventions (pharmacological or non-pharmacological) or to an intervention and control arm. 


\section{Data abstraction and analysis}

Before starting data abstraction, we will conduct calibration exercises to ensure consistency between reviewers. Teams of reviewers will extract data independently and in duplicate from each eligible study using standardised forms and a detailed instruction manual to inform tailoring of an online data abstraction programme, DistillerSR (http:// systematic-review.net/). We will extract data regarding patient demographics, trial methodology, intervention details and outcome data guided by the Initiative on Methods, Measurement and Pain Assessment in Clinical Trials (IMMPACT) ${ }^{62}{ }^{63}$ Specifically, we will collect outcome data across the following nine IMMPACT-recommended core outcome domains: (1) pain; (2) physical functioning; (3) emotional functioning; (4) participant ratings of improvement and satisfaction with treatment; (5) symptoms and adverse events; (6) participation disposition; (7) role functioning; (8) interpersonal functioning; and (9) sleep and fatigue. We will collect data for all adverse outcomes as guided by Ioannidis and Lau. ${ }^{64}$ We will resolve disagreements by discussion to achieve consensus.

\section{Evaluating risk of bias in individual studies}

Reviewers will assess risk of bias using a modified Cochrane risk of bias instrument that includes response options of 'definitely or probably yes'-assigned a low risk of bias-or 'definitely or probably no'-assigned a high risk of bias, an approach we have previously shown to be valid. ${ }^{65}$ We will evaluate sequence generation, allocation sequence concealment; blinding of participants and study personnel; and incomplete outcome data. ${ }^{66}$ We will resolve any disagreements between reviewers by discussion. We will contact study authors if limitations in reporting lead to uncertainties in eligibility, risk of bias, or outcome.

\section{Direct comparisons meta-analyses}

In comparison to fixed effect models, random effect models are conservative in that they consider the withinstudy as well as the among-study variability. Recent methodological research has shown that while popular, the DerSimonian-Laird method ${ }^{67}$ can produce narrow CIs when the number of studies is small or when they are substantively heterogeneous. ${ }^{68} 69$ Therefore, to pool outcome data for trials that make direct comparisons between interventions and alternatives, we will use the likelihood profile approach. ${ }^{70}$ We will pool cross-over trials with parallel design RCTs using methods outlined in the Cochrane handbook to derive effect estimates. ${ }^{66}$ Specifically, we will perform a paired t test for each crossover trial if any of the following are available: (1) the individual participant data; (2) the mean and SD or SE of the participant-specific differences, and between the intervention and control measurement; (3) the mean difference (MD) and one of the following: (a) a t-statistic from a paired t test; (b) a $\mathrm{p}$ value from a paired t-test; (c) a CI from a paired analysis; or (4) a graph of measurements of the intervention arm and control arm from which we can extract individual data values, so long as the matched measurement for each individual can be identified. ${ }^{66}$ If these data are not available, we will approximate paired analyses by calculating the MDs and the corresponding SEs for the paired analyses. ${ }^{66}$ If the SE or SD of withinparticipant differences are not available, we will impute the SD using the methods outlined in the Cochrane Handbook. ${ }^{66}$

\section{Ensuring interpretable results}

We will use a number of approaches to provide interpretable results from our meta-analyses. For studies that provide binary outcome measures, we will calculate relative risks (RRs) to inform relative effectiveness. To generate measures of absolute effect (risk differences), we will use estimates of baseline risk from the control arm of eligible RCTs.

When pooling across studies reporting continuous endpoints that use the same instrument, we will calculate the weighted mean difference (WMD), which maintains the original unit of measurement and represents the average difference between groups. Once the WMD has been calculated, we will contextualise this value by noting the corresponding MID-the smallest change in instrument score that patients perceive is important. We will prioritise use of anchor-based MIDs when they are available, and calculate distribution-based MIDs when they are not. We will also divide WMDs by their corresponding MID to obtain estimates in MID units. However, contextualising the WMD through the MID can be misleading; clinicians may mistakenly interpret any effect in MID units smaller than 1 as suggesting no patient obtains an important benefit, and any effect estimate greater than 1 as suggesting that all patients benefit, which is not accurate. Therefore, we will also calculate the proportion of patients who have benefited, that is, demonstrated improvement greater than or equal to the MID in each trial, then aggregate the results across all studies. ${ }^{71}$ Further, we will convert the proportion data to probabilities of experiencing benefit to calculate pooled RRs and numbers needed to treat (NNTs).

For trials using different continuous outcome measures that address the same underlying construct, we will calculate the between-group difference in change scores (change from baseline) and divide this difference by the SD of the change. This calculation creates a measure of the effect (quantifying its magnitude in SD units), called the standardised mean difference (SMD), which allows for comparison and pooling across trials. ${ }^{66}$ However, the SMD is difficult to interpret and is vulnerable to the heterogeneity of patients who are enrolled: trials that enrol homogeneous study populations and thus have smaller SDs will generate a larger SMD than studies with more heterogeneous patient populations. To address this issue, we will calculate the effect estimates in MID units by dividing between-group difference in change scores by the MID. However, as with WMDs, contextualising the SMD in MID units can be misleading; therefore, we will, 
for each trial, calculate the probability of experiencing a treatment effect greater than or equal to the MID in the control and intervention groups, then pool the results to calculate RRs and NNTs. ${ }^{71}$

Patients may be interested in the ability of a given intervention to provide more than an MID-to produce improvement that allows patients to feel much better (ie, substantially greater than the MID). Thus, for our analyses, where studies report percentage reduction in pain we will also use thresholds of $\geq 20 \%, \geq 30 \%$ and $\geq 50 \%$ reduction of pain from baseline to calculate the proportion of patients who have benefited in each trial, and derive RRs and risk differences.

\section{Assessment of heterogeneity and subgroup analyses}

We will conduct conventional meta-analyses (see above) for each paired comparison. For each of these comparisons, we will examine heterogeneity using a $\chi^{2}$ test and the $\mathrm{I}^{2}$ statistic-the percentage of variability that is due to true differences between studies (heterogeneity) rather than sampling error (chance). ${ }^{72} 73$

We have generated five a priori hypotheses to explain variability between studies: (1) subjective syndromes will show smaller treatment effects versus objectively diagnosed syndromes; (2) trials comparing treatment to placebo will show larger treatment effects than trials using active comparators; (3) trials that exclude patients who are receiving disability benefits and/or involved in litigation will show larger treatment effects than trials that include such patients; (4) chronic neuropathic pain syndromes defined by peripheral nervous system lesions (eg, diabetic neuropathy) will show larger effects than central nervous system lesions (eg, chronic post-stroke pain); (5) trials with higher risk of bias will show larger treatment effects than trials with lower risk of bias; and (6) trials with longer follow-up times will show smaller treatment effects than trials with shorter follow-up times. To inform our subgroup analyses based on risk of bias we will, if we detect variability within the individual risk of bias components, perform subgroup analyses on a component-by-component basis. We will perform meta-regression and subgroup analyses to explore these hypotheses, and interpret the results in the context of the GRADE system (see below). ${ }^{74}$

\section{Confidence in the estimates of effect}

We will use the GRADE approach to evaluate confidence in effect estimates for all reported outcomes. ${ }^{75}$ GRADE has been adopted by over 70 organisations worldwide, and this approach facilitates transparent, rigorous and comprehensive assessment of evidence quality on a per outcome basis. ${ }^{76-89}$ Our review of the management of chronic neuropathic pain will be the first to use the GRADE criteria to evaluate confidence in effect estimates. We will categorise the confidence in estimates (quality of evidence) as high, moderate, low or very low. Using this approach, randomised trials begin as high quality evidence but may be rated down by one or more of four categories of limitations. We will use GRADE guidance to determine whether to rate down confidence in the body of evidence for (1) risk of bias ${ }^{87}$ and for (2) imprecision, ${ }^{81}$ inconsistency ${ }^{83}$ and publication bias. ${ }^{84}$ For the risk of bias assessment, for any comparisons that suggest a statistically significant treatment effect, we will use recently developed approaches to address missing participant data for dichotomous outcomes and continuous outcomes. ${ }^{90}{ }^{91}$ When plausible worst case scenarios reverse the treatment effect we will rate down for risk of bias. We will present the results of our meta-analyses in GRADE evidence profiles that will provide a succinct, easily digestible presentation of the risk of bias and magnitude of effects. ${ }^{75}$

\section{Multiple treatment comparison meta-analyses}

To assess relative effects of competing treatments, we will construct a random effects model within the Bayesian framework using Markov chain Monte Carlo methods. ${ }^{92}$ We will use trace plots and calculate the Gelman-Rubin statistic to assess model convergence. We will model patient-important outcomes in every treatment group of every study, and specify the relations among the effect sizes across studies. ${ }^{93}$ This method combines direct and indirect evidence for any given pair of treatments. We will use the resulting $95 \%$ credible intervals to assess the precision of treatment effects. ${ }^{94}$ A key assumption behind multiple treatment comparison meta-analysis is that the analysed network is consistent or coherent, that is, that direct and indirect evidence on the same comparisons do not disagree beyond chance. We will identify and estimate incoherence by employing a mixed treatment comparisons incoherence model in the Bayesian framework. ${ }^{95}$ For each comparison, we will note the direct estimates and associated CIs from the previous analysis and calculate the indirect estimate using a node splitting procedure as well as the network estimate. We will conduct a statistical test for incoherence between the direct and the indirect estimate.

We will have assessed confidence in estimates of effect from the direct comparisons in our pair-wise meta-analyses described previously. For rating confidence in the indirect comparisons, we will focus our assessments on first-order loops (ie, loops that are connected to the interventions of interest through only one other intervention; eg, A vs C and $B$ vs $C$ to estimate effects of A vs B) with the lowest variances, and thus contribute the most to the estimates of effect. Within each loop, our confidence in the indirect comparison will be the lowest of the confidence ratings we have assigned to the contributing direct comparisons. For instance, if treatment $A$ versus $C$ warrants high confidence and $\mathrm{B}$ versus $\mathrm{C}$ warrants moderate confidence, we will judge the associated indirect comparison (A vs B) as warranting moderate confidence. We may rate down confidence in the indirect comparisons further if we have a strong suspicion that the transitivity assumption (ie, the assumption that there are no effect modifiers-such as differences in patients, extent to which interventions have 
been optimally administered, differences in the comparator, and differences in how the outcome has been measured-in the two direct comparisons that may bias the indirect estimate) has been violated.

Our overall judgement of confidence in the network estimate for any paired comparison will be the higher of the confidence rating among the contributing direct and indirect comparisons. However, we may rate down confidence in the network estimate if we find that the direct and indirect estimates are incoherent.

As a secondary analysis, we will rank the interventions using the SUCRA (surface under the cumulative ranking) method. ${ }^{96}$ The SUCRA rankings may be misleading: if there is only evidence warranting low confidence for most comparisons; if the evidence supporting the higher ranked interventions warrants lower confidence than the evidence supporting the lower ranked interventions; or if the magnitude of effect is very similar in higher versus lower ranked comparisons. We will consider these issues in interpreting the SUCRA rankings.

\section{DISCUSSION}

With the established high prevalence of chronic neuropathic pain worldwide, the associated high socioeconomic burden and the paucity of evidence on the comparative effectiveness of treatment options, there is an urgent and critical need for a high-quality systematic review to inform evidence-based management of chronic neuropathic pain.

Our proposed review has several strengths in relation to existing reviews. First, we will include all nonpharmacological and pharmacological treatment options for all chronic neuropathic pain syndromes. It is plausible that individual pain syndromes, in general, respond similarly to similar interventions, and thus by pooling across individual syndromes, it may be possible to provide a more precise estimate of treatment effect. In addition, examining all therapies for all chronic neuropathic pain syndromes would provide comprehensive guidance for management of chronic neuropathic pain, which increases utility to healthcare providers, patients and payers. Second, we will update the search to present date, explore a wider range of literature databases than existing reviews and include eligible articles in all languages. Third, we will make all subjective decisions, including determining trial eligibility and collecting data, in teams of reviewers, independently and in duplicate, with assessments of the reproducibility of judgements. Fourth, we will focus on collecting patient-important outcomes across IMMPACT-recommended core domains. Fifth, we will use the GRADE approach to evaluate our confidence in treatment effects. Sixth, we will ensure interpretability by presenting risk differences and measures of relative effect for all outcomes reported, and by presenting our findings with GRADE evidence profiles. Seventh, we will generate a limited number of a priori subgroup hypotheses to explain heterogeneity of pooled estimates of treatment effect, and conduct meta-regression and subgroup analyses consistent with best current practices.

As with existing reviews, the results of our proposed systematic review will be limited by possible shortcomings of the primary studies, including presence of publication bias, high heterogeneity, and poor quality of reporting and methodological rigour. Another likely limitation, unique to multiple treatment comparison meta-analyses, will be the nature of available treatment comparisons to build robust networks for our analyses.

The findings of our review will help inform patients with chronic neuropathic pain about their therapeutic options, so that they can make more autonomous health management decisions. In addition, to help educate clinicians responsible for managing such patients, our review will facilitate updating clinical practice guidelines for the management of chronic neuropathic pain.

\section{Author affiliations}

${ }^{1}$ Department of Clinical Epidemiology \& Biostatistics, McMaster University, Hamilton, Ontario, Canada

${ }^{2}$ Outcomes Research Consortium, Cleveland Clinic, Cleveland, Ohio, USA

${ }^{3}$ Department of Anesthesia, McMaster University, Hamilton, Ontario, Canada

${ }^{4}$ Michael G. DeGroote Institute for Pain Research and Care, McMaster

University, Hamilton, Ontario, Canada

${ }^{5}$ Departments of Clinical Neurological Sciences and Oncology, Western University, London, Ontario, Canada

${ }^{6}$ Department of Medicine, McMaster University, Hamilton, Ontario, Canada ${ }^{7}$ Department of Outcomes Research, Cleveland Clinic, Cleveland, Ohio, USA ${ }^{8}$ Knowledge and Evaluation Research Unit, Divisions of Endocrinology and Diabetes, and Health Care \& Policy Research, Mayo Clinic, Rochester, Minnesota, USA

${ }^{9}$ Department of Pediatrics, McMaster University, Hamilton, Ontario, Canada

Contributors All authors made substantial contributions to conception and design. SMM drafted the article, and DNB, DEM, RC, ZI, AA, AP, LW, SMK, AT, VMM, DIS, LT, GHG and JWB revised it critically for important intellectual content. All authors provided final approval of the version to be published.

Funding This systematic review is supported by the Canadian Anesthesia Research Foundation and the Canadian Institutes of Health Research.

Competing interests DEM and AP are chair and member, respectively, of the Canadian Pain Society Guideline Committee for management of chronic neuropathic pain. DEM has received research grant funding from Pfizer Canada, and has received honoraria for educational presentations from Jansenn-Ortho, Lilly, Purdue Pharma and Merck-Frosst.

Provenance and peer review Not commissioned; externally peer reviewed.

Open Access This is an Open Access article distributed in accordance with the Creative Commons Attribution Non Commercial (CC BY-NC 4.0) license, which permits others to distribute, remix, adapt, build upon this work noncommercially, and license their derivative works on different terms, provided the original work is properly cited and the use is non-commercial. See: http:// creativecommons.org/licenses/by-nc/4.0/

\section{REFERENCES}

1. Treede RD, Jensen TS, Campbell J, et al. Neuropathic pain Redefinition and a grading system for clinical and research purposes. Neurology 2008;70:1630-5.

2. Baron R, Förster M, Binder A. Subgrouping of patients with neuropathic pain according to pain-related sensory abnormalities: a first step to a stratified treatment approach. Lancet Neurol 2012;11:999-1005.

3. Meyer H. Neuropathic pain-Current concepts. S Afr Fam Practice 2008;50:40-9.

4. Gilron I, Watson CPN, Cahill CM, et al. Neuropathic pain: a practica guide for the clinician. CMAJ 2006;175:265-75. 
5. O'Donnell MJ, Diener HC, Sacco RL, et al. Chronic pain syndromes after ischemic stroke: PRoFESS trial. Stroke 2013;44:1238-43.

6. Attal N. Neuropathic pain: mechanisms, therapeutic approach, and interpretation of clinical trials. Continuum (Minneapolis, Minn) 2012:18:161-75.

7. van Hecke O, Austin SK, Khan RA, et al. Neuropathic pain in the general population: a systematic review of epidemiological studies. Pain 2014;155:654-62.

8. Doth AH, Hansson PT, Jensen MP, et al. The burden of neuropathic pain: a systematic review and meta-analysis of health utilities. Pain 2010;149:338-44

9. McDermott AM, Toelle TR, Rowbotham DJ, et al. The burden of neuropathic pain: results from a cross-sectional survey. Eur $J$ Pain 2006;10:127-35.

10. Attal N, Lanteri-Minet M, Laurent B, et al. The specific disease burden of neuropathic pain: results of a French nationwide survey. Pain 2011;152:2836-43.

11. Langley PC, Van Litsenburg C, Cappelleri JC, et al. The burden associated with neuropathic pain in Western Europe. J Med Econ 2013;16:85-95

12. Tarride J, Collet $\mathrm{J}$, Choiniere $\mathrm{M}$, et al. The economic burden of neuropathic pain in Canada. J Med Econ 2006;9:55-68.

13. Berger A, Dukes EM, Oster G. Clinical characteristics and economic costs of patients with painful neuropathic disorders. J Pain 2004;5:143-9.

14. Simpson EL, Duenas A, Holmes MW, et al. Spinal cord stimulation for chronic pain of neuropathic or ischaemic origin: systematic review and economic evaluation. Health Technol Assess 2009;13:iii, ix-x, $1-154$.

15. Liu $\mathrm{H}, \mathrm{Li} \mathrm{H}, \mathrm{Xu} \mathrm{M}$, et al. A systematic review on acupuncture for trigeminal neuralgia. Altern Ther Health Med 2010;16:30-5.

16. Pittler MH, Ernst E. Complementary therapies for neuropathic and neuralgic pain: systematic review. Clin J Pain 2008;24:731-3.

17. Wetering EJ, Lemmens KM, Nieboer AP, et al. Cognitive and behavioral interventions for the management of chronic neuropathic pain in adults--a systematic review. Eur J Pain 2010;14:670-81.

18. Saarto T, Wiffen PJ. Antidepressants for neuropathic pain. Cochrane Database Syst Rev 2007:CD005454.

19. Goodyear-Smith F, Halliwell J. Anticonvulsants for neuropathic pain: gaps in the evidence. Clin J Pain 2009;25:528-36.

20. Eisenberg E, McNicol E, Carr DB. Opioids for neuropathic pain. Cochrane Database Syst Rev 2006;(3):CD006146.

21. Sauzet O, Williams JE, Ross J, et al. The characteristics and quality of randomized controlled trials in neuropathic pain: a descriptive study based on a systematic review. Clin J Pain 2013;29:591-9.

22. Hearn L, Derry S, Moore RA. Lacosamide for neuropathic pain and fibromyalgia in adults. Cochrane Database Syst Rev 2012;2: CD009318.

23. Derry S, Moore RA. Topical capsaicin (low concentration) for chronic neuropathic pain in adults. Cochrane Database Syst Rev 2012;9: CD010111.

24. Gill D, Derry S, Wiffen PJ, et al. Valproic acid and sodium valproate for neuropathic pain and fibromyalgia in adults. Cochrane Database Syst Rev 2011;(10):CD009183.

25. Moore RA, Wiffen PJ, Derry S, et al. Gabapentin for chronic neuropathic pain and fibromyalgia in adults. Cochrane Database Syst Rev 2011;(3):CD007938.

26. Wiffen PJ, Derry S, Moore RA, et al. Carbamazepine for acute and chronic pain in adults. Cochrane Database Syst Rev 2011;(1): CD005451.

27. Chen N, Yang M, He L, et al. Corticosteroids for preventing postherpetic neuralgia. Cochrane Database Syst Rev 2010;(12): CD005582.

28. Collins S, Sigtermans MJ, Dahan A, et al. NMDA receptor antagonists for the treatment of neuropathic pain. Pain Med 2010;11:1726-42.

29. Lunn MP, Hughes RA, Wiffen PJ. Duloxetine for treating painful neuropathy or chronic pain. Cochrane Database Syst Rev 2009;(4): CD007115.

30. Leung A, Donohue M, Xu R, et al. rTMS for suppressing neuropathic pain: a meta-analysis. J Pain 2009;10:1205-16.

31. Khaliq W, Alam S, Puri N. Topical lidocaine for the treatment of postherpetic neuralgia. Cochrane Database Syst Rev 2007;(2): CD004846.

32. Vargas-Espinosa ML, Sanmarti-Garcia G, Vazquez-Delgado E, et al. Antiepileptic drugs for the treatment of neuropathic pain: a systematic review. Med Oral Patol Oral Cir Bucal 2012;17:e786-93.

33. Tremont-Lukats IW, Challapalli V, McNicol ED, et al. Systemic administration of local anesthetics to relieve neuropathic pain: a systematic review and meta-analysis. Anesth Analg $2005 ; 101: 1738-49$
34. Chaparro LE, Wiffen PJ, Moore RA, et al. Combination pharmacotherapy for the treatment of neuropathic pain in adults. Cochrane Database Syst Rev 2012;7:CD008943.

35. Derry S, Sven-Rice A, Cole P, et al. Topical capsaicin (high concentration) for chronic neuropathic pain in adults. Cochrane Database Syst Rev 2013;2:CD007393.

36. Gaskell H, Moore RA, Derry S, et al. Oxycodone for neuropathic pain and fibromyalgia in adults. Cochrane Database Syst Rev 2014;6:CD010692.

37. Hearn L, Derry S, Phillips T, et al. Imipramine for neuropathic pain in adults. Cochrane Database Syst Rev 2014;5:CD010769.

38. Moore RA, Derry S, Aldington D, et al. Amitriptyline for neuropathic pain and fibromyalgia in adults. Cochrane Database Syst Rev 2012;12:CD008242.

39. Moore RA, Wiffen PJ, Derry S, et al. Gabapentin for chronic neuropathic pain and fibromyalgia in adults. Cochrane Database Syst Rev 2014;4:CD007938.

40. Straube S, Derry S, Moore RA, et al. Cervico-thoracic or lumbar sympathectomy for neuropathic pain and complex regional pain syndrome. Cochrane Database Syst Rev 2013;9:CD002918.

41. Wiffen PJ, Derry S, Lunn MP, et al. Topiramate for neuropathic pain and fibromyalgia in adults. Cochrane Database Syst Rev 2013;8: CD008314.

42. Wiffen PJ, Derry S, Moore RA. Lamotrigine for chronic neuropathic pain and fibromyalgia in adults. Cochrane Database Syst Rev 2013;12:CD006044.

43. Wiffen PJ, Derry S, Moore RA, et al. Antiepileptic drugs for neuropathic pain and fibromyalgia-an overview of Cochrane reviews. Cochrane Database Syst Rev 2013;11:CD010567.

44. Wiffen PJ, Derry S, Moore RA, et al. Carbamazepine for chronic neuropathic pain and fibromyalgia in adults. Cochrane Database Syst Rev 2014;4:CD005451

45. Wiffen PJ, Derry S, Moore RA, et al. Levetiracetam for neuropathic pain in adults. Cochrane Database Syst Rev 2014;7:CD010943.

46. Derry S, Wiffen PJ, Moore RA, et al. Topical lidocaine for neuropathic pain in adults. Cochrane Database Syst Rev 2014;7: CD010958.

47. Edelsberg JS, Lord C, Oster G. Systematic review and meta-analysis of efficacy, safety, and tolerability data from randomized controlled trials of drugs used to treat postherpetic neuralgia. Ann Pharmacother 2011;45:1483-90.

48. Zakrzewska JM, Akram H. Neurosurgical interventions for the treatment of classical trigeminal neuralgia. Cochrane Database Syst Rev 2011;(9):CD007312.

49. Yang M, Zhou M, He L, et al. Non-antiepileptic drugs for trigeminal neuralgia. Cochrane Database Syst Rev 2011;(1):CD004029.

50. van Alfen N, van Engelen BG, Hughes RA. Treatment for idiopathic and hereditary neuralgic amyotrophy (brachial neuritis). Cochrane Database Syst Rev 2009;(3):CD006976.

51. Griebeler ML, Tsapas A, Brito JP, et al. Pharmacologic interventions for painful diabetic neuropathy: an umbrella systematic review and comparative effectiveness network meta-analysis (Protocol). Syst rev 2012;1:61.

52. Wong MC, Chung JW, Wong TK. Effects of treatments for symptoms of painful diabetic neuropathy: systematic review. BMJ 2007;335:87.

53. Snedecor SJ, Sudharshan L, Cappelleri JC, et al. Systematic review and meta-analysis of pharmacological therapies for painful diabetic peripheral neuropathy. Pain Pract 2013;14:167-84.

54. Xia L, Zhong J, Zhu J, et al. Effectiveness and safety of microvascular decompression surgery for treatment of trigeminal neuralgia: a systematic review. J Craniofac Surg 2014;25:1413-17.

55. Rudroju N, Bansal D, Talakokkula ST, et al. Comparative efficacy and safety of six antidepressants and anticonvulsants in painful diabetic neuropathy: a network meta-analysis. Pain Physician 2013;16:E705-14.

56. Ney JP, Devine EB, Watanabe JH, et al. Comparative efficacy of ora pharmaceuticals for the treatment of chronic peripheral neuropathic pain: meta-analysis and indirect treatment comparisons. Pain Med 2013;14:706-19.

57. Selph S, Carson S, Fu R, et al. Drug class review: neuropathic pain: final update 1 report. Portland, OR: Oregon Health \& Science University, 2011.

58. Berger VW. Is the Jadad score the proper evaluation of trials? J Rheumatol 2006;33:1710-1; author reply 1711-1712.

59. Systematic Review of Quality Assessment Instruments for Randomized Control Trials: Selection of SIGN50 Methodological Checklist. http://www.imbi.uni-freiburg.de/OJS/cca/index.php/cca/ article/view/5053

60. Busse JW, Bruno P, Malik K, et al. An efficient strategy allowed English-speaking reviewers to identify foreign-language articles eligible for a systematic review. J Clin Epidemiol 2014;67:547-53. 
61. Landis JR, Koch GG. The measurement of observer agreement for categorical data. Biometrics 1977;33:159-74.

62. Turk DC, Dworkin RH, Allen RR, et al. Core outcome domains for chronic pain clinical trials: IMMPACT recommendations. Pain 2003;106:337-45.

63. Turk DC, Dworkin RH, Revicki D, et al. Identifying important outcome domains for chronic pain clinical trials: an IMMPACT survey of people with pain. Pain 2008;137:276-85.

64. Ioannidis JP, Lau J. Completeness of safety reporting in randomized trials: an evaluation of 7 medical areas. JAMA 2001;285:437-43.

65. Akl EA, Sun X, Busse JW, et al. Specific instructions for estimating unclearly reported blinding status in randomized trials were reliable and valid. J Clin Epidemiol 2012;65:262-7.

66. Higgins JP, Green S. Cochrane Handbook for Systematic Reviews of Interventions, Version [5.1.0] (updated March 2011).

The Cochrane Collaboration, 2011

67. DerSimonian R, Laird N. Meta-analysis in clinical trials. Control Clin Trials 1986;7:177-88.

68. Cornell JE, Mulrow CD, Localio R, et al. Random-effects meta-analysis of inconsistent effects: a time for change. Ann Intern Med 2014;160:267-70.

69. Brockwell SE, Gordon IR. A comparison of statistical methods for meta-analysis. Stat med 2001;20:825-40.

70. Guolo A. Higher-order likelihood inference in meta-analysis and meta-regression. Stat med 2012;31:313-27.

71. Thorlund K, Walter SD, Johnston BC, et al. Pooling health-related quality of life outcomes in meta-analysis-a tutorial and review of methods for enhancing interpretability. Res Synth Methods 2011;2:188-203.

72. Higgins JP, Thompson SG. Quantifying heterogeneity in a meta-analysis. Stat Med 2002;21:1539-58.

73. Higgins JP, Thompson SG, Deeks JJ, et al. Measuring inconsistency in meta-analyses. BMJ 2003;327:557-60.

74. Altman DG, Bland JM. Interaction revisited: the difference between two estimates. BMJ 2003;326:219.

75. Atkins $D$, Best $D$, Briss PA, et al. Grading quality of evidence and strength of recommendations. BMJ 2004;328:1490.

76. Balshem $\mathrm{H}$, Helfand $\mathrm{M}$, Schunemann $\mathrm{HJ}$, et al. RADE guidelines: 3. Rating the quality of evidence. J Clin Epidemiol 2011;64:401-6.

77. Brunetti M, Shemilt I, Pregno S, et al. GRADE guidelines: 10. Considering resource use and rating the quality of economic evidence. J Clin Epidemiol 2013:66:140-50.

78. Guyatt G, Oxman AD, Akl EA, et al. GRADE guidelines: 1. Introduction-GRADE evidence profiles and summary of findings tables. J Clin Epidemiol 2011;64:383-94.

79. Guyatt G, Oxman AD, Sultan S, et al. GRADE guidelines: 11. Making an overall rating of confidence in effect estimates for a single outcome and for all outcomes. J Clin Epidemiol 2013;66:151-7.
80. Guyatt GH, Oxman AD, Kunz R, et al. GRADE guidelines: 2. Framing the question and deciding on important outcomes. J Clin Epidemiol 2011;64:395-400.

81. Guyatt GH, Oxman AD, Kunz R, et al. GRADE guidelines 6. Rating the quality of evidence-imprecision. $J$ Clin Epidemiol 2011;64:1283-93.

82. Guyatt GH, Oxman AD, Kunz R, et al. GRADE guidelines: 8. Rating the quality of evidence-indirectness. J Clin Epidemiol 2011;64:1303-10.

83. Guyatt GH, Oxman AD, Kunz R, et al. GRADE guidelines: 7. Rating the quality of evidence-inconsistency. J Clin Epidemiol 2011;64:1294-302.

84. Guyatt GH, Oxman AD, Montori V, et al. GRADE guidelines: 5. Rating the quality of evidence-publication bias. J Clin Epidemiol 2011;64:1277-82.

85. Guyatt GH, Oxman AD, Santesso N, et al. GRADE guidelines: 12. Preparing summary of findings tables-binary outcomes. J Clin Epidemiol 2013;66:158-72.

86. Guyatt $\mathrm{GH}$, Oxman AD, Sultan $\mathrm{S}$, et al. GRADE guidelines: 9. Rating up the quality of evidence. $J$ Clin Epidemiol 2011;64:1311-16.

87. Guyatt GH, Oxman AD, Vist G, et al. GRADE guidelines: 4. Rating the quality of evidence--study limitations (risk of bias). J Clin Epidemiol 2011;64:407-15.

88. Guyatt GH, Thorlund K, Oxman AD, et al. GRADE guidelines: 13. Preparing summary of findings tables and evidence profiles-continuous outcomes. J Clin Epidemiol 2013;66:173-83.

89. Andrews J, Guyatt G, Oxman AD, et al. GRADE guidelines: 15 . Going from evidence to recommendations: the significance and presentation of recommendations. J Clin Epidemiol 2013;66:719-25.

90. Ebrahim S, Akl EA, Mustafa RA, et al. Addressing continuous data for participants excluded from trial analysis: a guide for systematic reviewers. J Clin Epidemiol 2013;66:1014-21.e1.

91. Akl EA, Johnston BC, Alonso-Coello $\mathrm{P}$, et al. Addressing dichotomous data for participants excluded from trial analysis: a guide for systematic reviewers. PLOS ONE 2013;8:e57132.

92. Ades AE, Sculpher M, Sutton A, et al. Bayesian methods for evidence synthesis in cost-effectiveness analysis. Pharmacoeconomics 2006;24:1-19.

93. Salanti G, Higgins JP, Ades AE, et al. Evaluation of networks of randomized trials. Stat Methods Med Res 2008;17:279-301.

94. Sterne JA, Davey Smith G. Sifting the evidence-what's wrong with significance tests? BMJ 2001;322:226-31.

95. Lu G, Ades A. Assessing evidence inconsistency in mixed treatment comparisons. J Stat Assoc 2006;101:447-59.

96. Salanti G, Ades AE, loannidis JP. Graphical methods and numerical summaries for presenting results from multiple-treatment metaanalysis: an overview and tutorial. J Clin Epidemiol 2011;64:163-71. 